\title{
Clinical Application and Progress of Fecal Microbiota Transplantation in Liver Diseases: A Review
}

\author{
Xinpei Gu, MD ${ }^{1}$ Qin Lu, MD ${ }^{2}$ Chengcheng Zhang, \\ ${ }^{1}$ Department of Human Anatomy, Shandong First Medical University \\ and Shandong Academy of Medical Sciences, Taian, China \\ 2 Department of Prescription Science, School of Basic Medical \\ Sciences, Hebei University of Chinese Medicine, Shijiazhuang, China \\ ${ }^{3}$ Department of Medical Oncology, Shuguang Hospital, Shanghai \\ University of Traditional Chinese Medicine, Shanghai, China \\ ${ }^{4}$ Institute of Child Development and Education, School of Biological \\ Sciences and Medical Engineering, Southeast University, Nanjing, China \\ Semin Liver Dis 2021;41:495-506.
}

\author{
Address for correspondence Liuxi Chu, PhD, Institute of Child \\ Development and Education, School of Biological Sciences and \\ Medical Engineering, Southeast University, Nanjing - 210096, China \\ (e-mail: clx@seu.edu.cn).
}

\begin{abstract}
Keywords

- gut microbiota

- microbiota imbalance

- liver diseases

- microbiome

The human gut harbors a dense and highly diverse microbiota of approximately 1,000 bacterial species. The interaction between the host and gut bacteria strongly influences human health. Numerous evidence suggest that intestinal flora imbalance is closely associated with the development and treatment of liver diseases, including acute liver injury and chronic liver diseases (cirrhosis, autoimmune liver disease, and fatty liver). Therefore, regulating the gut microbiota is expected to be a new method for the adjuvant treatment of liver diseases. Fecal microbiota transplantation (FMT) is defined as the transplantation of gut microbiota from healthy donors to sick patients via the upper or lower gastrointestinal route to restore the normal intestinal balance. In this study, we briefly review the current research on the gut microbiota and its link to liver diseases and then summarize the evidence to elucidate the clinical application and development of FMT in liver disease treatment.
\end{abstract}

The human microbiome is defined as a collection of microbes -bacteria, viruses, fungi-that colonize the human body. Microbial cells in the body are as abundant as our somatic cells. The gut, the largest digestive and immune organ, contains approximately $80 \%$ of the total microbiota in humans. ${ }^{1}$ More than $99 \%$ of the microbes in the human gut are bacteria and other microbes, such as viruses and fungi. ${ }^{2}$ The gut contains approximately 1,000 bacterial species, with 2,000 genes per species. There are two million genes in the gut, which is 100 times higher than the commonly estimated number of human genes (approximately 20,000). Some of these bacteria are probiotic, while some are pathogenic. The dynamic balance of the number and species of bacteria in the gut maintains the intestinal balance, which plays an important role in the digestive, metabolic, and immune functions of the human body.

published online July 14,2021
DOI https://doi.org/ 10.1055/s-0041-1732319. ISSN 0272-8087.
There are many factors affecting the intestinal balance, such as diet, medications, infections, metabolic disorders, and autoimmune diseases. An imbalance in the intestinal flora is related to the occurrence and development of many intestinal and extraintestinal diseases, including liver diseases, diabetes, leukemia, and sepsis. Therefore, altering the gut microbiota has the potential to combat diseases associated with intestinal dysbiosis. Many approaches have been employed to modulate the structure and metabolism of the gut microbiota, such as diet, prebiotics, probiotics, and antibiotics. $^{3,4}$ Although the above methods obtained certain curative effects, the results were not very satisfactory.

Fecal microbiota transplantation (FMT) may be traced back as early as the Eastern Jin Dynasty in China approximately 1,700 years ago. However, it has attracted the interest of clinical doctors and scholars only in recent
(C) 2021. The Author(s).

This is an open access article published by Thieme under the terms of the Creative Commons Attribution-NonDerivative-NonCommercial-License, permitting copying and reproduction so long as the original work is given appropriate credit. Contents may not be used for commercial purposes, or adapted, remixed, transformed or built upon. (https://creativecommons.org/ licenses/by-nc-nd/4.0/)

Thieme Medical Publishers, Inc., 333 Seventh Avenue, 18th Floor, New York, NY 10001, USA 
years. FMT is regarded as a promising treatment regimen to regulate gut microbiota directly. This method transfers processed fecal materials from healthy donors to patients to alter the composition of their gut microbiota. It has been widely accepted that FMT can successfully treat refractory and recurrent Clostridium difficile infection (CDI). Moreover, an increasing amount of data indicate that FMT is effective at ameliorating intractable functional constipation, inflammatory bowel diseases (IBDs), obesity, type-2 diabetes, etc. ${ }^{5}$ In addition, the observed intestinal dysbiosis that occurs in a variety of liver diseases has increased interest regarding the potential of FMT for the treatment of liver diseases.

The imbalance of gut microbiota is closely linked to the occurrence, development, and prognosis of several liver diseases, including acute liver injury, viral hepatitis, cirrhosis, autoimmune liver disease, alcoholic liver disease (ALD), and nonalcoholic fatty liver disease (NAFLD).${ }^{6,7}$ FMT has high efficacy in accelerating disease recovery by restoring the normal intestinal balance in patients with hepatic disorders. ${ }^{8,9}$ In this review, we mainly focus on the gut microbiota and its involvement in various liver diseases. We then discuss the current clinical studies and the latest advances in the application of FMT in acute and chronic liver diseases as shown in - Fig. 1.

\section{Connection between Gut Microbiota and Liver Diseases}

The liver, the largest digestive gland and an important immune organ in the human body, is in close contact with the gut. Studies have revealed that intestinal dysbiosis occurs in various liver diseases. ${ }^{6,7}$ Meanwhile, this dysbiosis influences the degree of hepatic steatosis, inflammation, fibrosis, and even canceration through multiple interactions with the host immune system and other cell types. The relationship between liver diseases and the gut microbiota is complex and not well understood. Liver diseases may lead to an imbalance in the gut microbiota, and this imbalance may aggravate the progression of liver diseases, which they mutually promote and affect.

\section{Changes in the Gut Microbiota Associated with Liver Disease}

The gut-liver axis, which was first described in 1987, proposes that the relationship between the gut and liver is bidirectional and a cyclic process. Under physiological conditions, the liver secretes bile acids (BAs) and other biologically active materials into the gut to aid in metabolism and absorption. During this process, in addition to liver and intestinal metabolites, endotoxins enter the liver with the

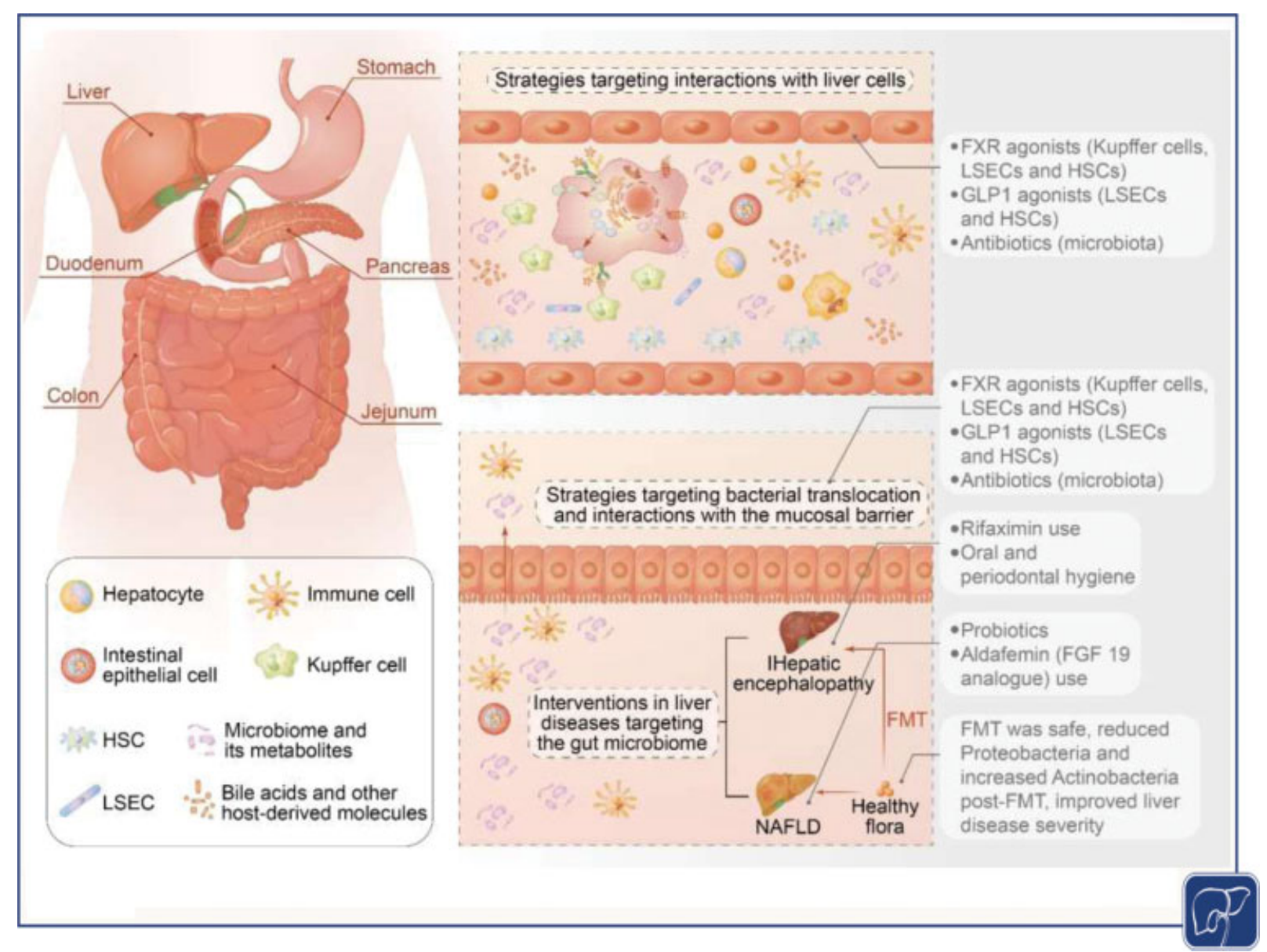

Fig. 1 Strategies and inventions to target the gut microbiome in liver diseases. Modulation of gut microbiota with treatments might improve the outcomes of patients with liver diseases. GLP1, Agonists for glucagon-like peptide 1; FXR, farnesoid X receptor; TGR5, Takeda G protein-coupled receptor 5; FGF19, fibroblast growth factor 19; HSC, hepatic stellate cell; LSEC, liver sinusoidal endothelial cell. 
blood via the portal system, affecting host systemic homeostasis. ${ }^{94,95}$

In healthy humans, the gut mainly consists of intestinal mucosal epithelium, intestinal mucus, and intestinal flora and serves a mucosal barrier function. The normal intestinal barrier could effectively limit the access of bacterial compounds. In disease conditions, such as liver diseases, the gut mucosal barrier could become damaged. Bacterial translocation (BT) from the gut to the systemic circulation or through the gut-liver axis could lead to the exacerbation of pathogenetic conditions.

NAFLD is a common clinical syndrome caused by factors other than alcohol consumption or any other well-established liver injury. Studies have suggested that alteration of the gut microbiota may be key to the development of NAFLD. As early as 20 years ago, it was reported that the application of prebiotics could counteract both fat mass development and hepatic steatosis. Le Roy et al demonstrated that the gut microbiota contributed to the development of NAFLD independently of obesity in germ-free mice. ${ }^{10}$ In addition, Gómez-Hurtado et $\mathrm{al}^{11}$ found that bacterial antigen translocation resulted in systemic inflammation in NAFLD patients.

Other liver diseases are also connected to changes in the composition of resident microbial communities. ${ }^{12}$ Studies have revealed gut microbiota dysbiosis in NAFLD patients, with lower median abundances of Bacteroidetes and Akkermansia muciniphila and higher abundances of Proteobacteria. ${ }^{13,14}$ Patients with primary biliary cholangitis also exhibit dysbiosis, with lower bacterial diversity observed than in healthy controls. Primary sclerosing cholangitis (PSC) patients showed increased levels of certain bacterial genera, such as Veillonella and Enterococcus, and loss of beneficial Clostridiales commensals. ${ }^{15}$ Compared with healthy individuals, patients with hepatitis B-related acute and chronic liver injury have increased circulating bacterial DNA and decreased microbial diversity. ${ }^{16}$ Gut-derived BT may be one of the causes of the increased peripheral bacterial load. In addition, a study by Wang et al showed that patients with liver cirrhosis have a unique gut microbiota composition, exhibiting an increase in Prevotella, Streptococcus, Staphylococcaceae, and Enterococcus and a decrease in Ruminococcus and Clostridium. ${ }^{17}$ In short, an imbalance in the gut microbiota has been observed in various liver diseases and may play a vital role in pathogenesis.

Several studies have identified specific metabolomics associated with different stage of the disease in NAFLD. ${ }^{18,19}$ Moreover, evidence of interaction among the gut, liver, and blood metabolites has emerged in the recent years, suggesting that the gut-liver axis is an important component in the development of NAFLD. Indeed, compositional changes in gut microbiota have been proposed to mechanistically contribute to the progression of NAFLD. ${ }^{20,21}$ Dysregulation of the gut microbiome has been implicated in the progression of NAFLD to advanced fibrosis and cirrhosis. The presence of advanced fibrosis in NAFLD is the most important predictor of liver mortality. Loomba et al used whole genome shotgun sequencing of DNA to analyze the patients with biopsy-proven NAFLD, of which 72 had mild/moderate (stage 0-2 fibrosis)
NAFLD, and 14 had advanced fibrosis (stage 3 or 4 fibrosis) and found that 37 bacterial species could distinguish mild/moderate NAFLD from advanced fibrosis, which could be used to better diagnose different courses of NAFLD. ${ }^{20}$ Moreover, the number of gut bacteria in patients with chronic liver disease has changed, increasing the possibility of using these changes to develop noninvasive diagnostic tools. Researchers from the Salk Institute for Biological Studies, Oh and their colleagues identified a feature in the gut microbiome that can detect liver cirrhosis caused by multiple causes in patients from different ethnicities and different geographic regions. ${ }^{22}$ In addition, it is possible to identify patients whose disease has progressed from fibrosis to cirrhosis. This study shows that the diagnostic stool test for liver cirrhosis has clinical and universal utility and emphasizes specific bacterial species as potential targets for novel therapeutic approaches. Recently, Oh and their colleagues reported that proton-pump inhibitor (PPI) use status did not modify the microbiome signature for cirrhosis. $^{23}$

\section{Effect of Microbiota Imbalance on the Development of Liver Disease}

Gut microbiota imbalance could exacerbate liver damage and be related to the progression of chronic hepatopathy, such as ALD, NAFLD, ${ }^{24-27}$ PBC, ${ }^{28-30}$ viral hepatitis, ${ }^{31}$ cirrhosis, ${ }^{17,27,32}$ and especially, hepatic encephalopathy (HE). ${ }^{33-35} \mathrm{HE}$ is a serious central nervous system complication of liver failure caused by different acute and chronic hepatitis or decompensated cirrhosis with significantly high mortality and morbidity. Currently, the precise mechanism of HE remains unclear. Among the several hypotheses, ammonia poisoning theory is the most recognized major pathogenesis. The elevated level of blood ammonia can promote the occurrence of HE and aggravate cerebral edema. This process is closely correlated with the imbalance of gut microbiota and variable gut-brain axes. ${ }^{36}$

The gut microbiota of cirrhosis patients with HE significantly differs from that of healthy individuals, such as the increase in Escherichia coli and Enterococcus. ${ }^{37}$ Bacterial blooms could result in an increase in the production of ammonia. At the same time, the imbalance of gut microbiota could damage mucosal barrier function, leading to increased intestinal mucosal permeability and bacterial endotoxin translocation. All of the above are risk factors for the development of HE. On the other hand, endotoxemia directly correlates with the development of liver failure, and leads to complications like HE. Likewise, endotoxemia plays an important role in the hyperdynamic circulation observed in patients with cirrhosis. ${ }^{38,39}$ Numerous studies have confirmed that gut-liver-brain axis can exert critical effects in HE via gut microflora, ${ }^{40,41}$ and the mechanism of $\mathrm{HE}$ has been associated with gut microflora. Indeed, the liver receives 75 to $80 \%$ of the hepatic blood volume via portal vein, and then the blood move into the system circulation. To prevent germs entering the bloodstream, intestinal barrier integrity can separate the luminal content from the internal milieu. Evidence has showed that ammonia and endotoxin were 
associated with the HE development in the setting of intestinal barrier dysfunction. ${ }^{42,43}$ Once destroyed, bacteria or bacterial products are transferred from tract to the liver. As the main metabolite of bacterial products, ammonia can produce potent hepatotoxicity. Meanwhile, neuroglial cells were activated by ammonia, and further release the proinflammatory factor. These factors lead to brain tissue damage followed by cognitive impairment. Furthermore, a series of preclinical and clinical studies have revealed bidirectional communication between the brain and the gut microbiota. It has been determined that gut microbes can send signals to the brain via neuronal, endocrine, and immunemediated pathways. The type and amount of gut microbial information that reaches the brain is greatly dependent on the regional intestinal microbes. Therefore, changes in the gut microbiota can impair brain function via the gut-brain axis.

Kang et $\mathrm{al}^{44}$ found that a dysbiotic profile of gut microbiota might be related to the development of systemic inflammation and neuroinflammation due to cirrhosis. The specific alterations of gut microbiota affect different aspects of brain function. This may explain the underlying mechanisms of HE from another perspective. Bajaj et $\mathrm{al}^{35}$ used 16S rRNA sequencing to analyze the stool/salivary microbiota of cirrhosis patients and found that the relative abundance of Lactobacillaceae was higher in minimal hepatic encephalopathy (MHE), which could be used to better diagnose MHE.

Caussy et $\mathrm{al}^{45}$ found that a gut microbiome-linked serum metabolite, 3-(4-hydroxyphenyl) lactate has a statistically and clinically significant shared gene-effect with both hepatic steatosis and fibrosis using two independent cohorts. This cross-sectional study provides evidence of a link between the gut-microbiome and 3-(4-hydroxyphenyl) lactate that shares gene effect with hepatic steatosis and fibrosis. Future studies from independent cohorts are needed to validate these findings and evaluate if 3-(4-hydroxyphenyl) lactate could be a useful noninvasive biomarker of gut microbiome involvement in NAFLD-related fibrosis and whether it could be used for monitoring the targeted therapeutic response when modulating the microbiome to affect hepatic fibrosis in NAFLD.

Gut microbiota abnormalities also affect acute liver injury (ALI). A previous study showed that BT and the translocation of endotoxin from the gut to the blood and intra-abdominal organs occurred in ALI induced by D-galactosamine (GalN). In acute alcohol-induced liver injury, a similar phenomenon was found: acute exposure to alcohol at high concentrations could cause dysbiosis of the gut microbiota and mucosal damage and then increase intestinal permeability, leading to the translocation of enteral bacteria and endotoxin from the intestine into the liver. Furthermore, the SCFAs derived from gut microbiota could strengthen $\mathrm{CD} 4^{+} \mathrm{T}$ cell-effector function during $\mathrm{T}$ cell-mediated acute inflammation, disrupting gut barrier function and damaging the liver. ${ }^{46}$ Lactobacillus rhamnosus GG was found to produce 5-methoxyindoleacetic acid to protect against oxidative liver injury by activating Nrf2. ${ }^{47}$

\section{Gut Microbiota and Liver Disease Treatment}

Extensive evidence supports the crucial role of altered communication among the gut, its microbiota, and the liver in the development of various acute and chronic liver diseases, as well as their complications. The imbalance of gut microbiota is a critical player in the pathophysiology of this process. In addition to the occurrence and development of liver disease, microbiota dysbiosis and BT also affect the ability to treat these diseases. In severe cirrhosis patients, an imbalance in the gut microbiota could lead to systemic inflammation, endotoxemia, and complications (spontaneous peritonitis, intestinal infection, etc.), ${ }^{48}$ which would limit therapeutic efficacy and may cause aggravation of the condition and even death. A clinical trial showed that patients with cirrhosis were often treated with antibiotics, which reduced the species and abundance of gut microbiota. This disturbed the gut microbiota composition and influenced the treatment result. ${ }^{49}$ Therefore, microbial factors are driving factors in many different liver diseases and at various stages of liver diseases. These findings have important therapeutic implications; we should place high value on the gut microbiota in the treatment of liver diseases. Manipulating the microbiota using various strategies, including prebiotics, new probiotics or antibiotics, or FMT, should be regarded as an important therapeutic measure. In view of the significant effect of FMT, it may become the most powerful measure to correct the imbalance of gut microbiota in the future.

\section{Fecal Microbiota Transplantation}

\section{Origin of Fecal Microbiota Transplantation}

FMT is an innovative clinical treatment that transfers screened and processed donor stool to a recipient via the upper or lower gastrointestinal route to restore a disrupted microbiota and ameliorate bacterial imbalances. Approximately 1,700 years ago in China, Ge Hong treated severe diarrhea by oral ingestion of fecal material. ${ }^{50}$ In 1958 , the administration of feces by enema to treat patients with pseudomembranous enterocolitis was reported. ${ }^{51}$ In 1983 , a patient with repeated episodes of Clostridium difficile enterocolitis was cured after achieving a normal bowel flora by feces infusion-the first clinical case of FMT. ${ }^{52}$ Later, FMT was widely used in CDI and other intestinal and extraintestinal diseases. Recent research attention has thus shifted toward its potential therapeutic role in acute and chronic liver disease.

\section{Fecal Microbiota Transplantation Methods}

Therapeutic protocols for FMT may vary significantly in different diseases, and donor selection is important. Healthy donors may be unrelated individuals, close relatives, or family members. However, the current guidelines recommend that the best donor source is an unrelated individual. This is because relatives and family members may share a common living environment and similar dietary structure. Therefore, they may share similar gut microbiota. By comparison, unrelated individuals have significantly different 
gut microbiota communities. To ensure successful transplantation, the donor needs to pass donor screening and meet strict inclusion and exclusion criteria according to an established protocol. ${ }^{5}$ Most stool materials are fresh, diluted, and homogenized to a form that can be administered. ${ }^{53}$ Frozen fecal materials can also be applied in some cases, which has the advantage of more convenient management. ${ }^{54}$ However, the bacterial diversity of frozen products may be lower than that of fresh material. Compared with fresh material, a recent double-blind study of patients with CDI reported that frozen fecal material provided lower therapeutic efficacy.

The routes of administration are also various. Fecal microbiota could be delivered to the gut through endoscopy, capsules, colonoscopy, enema and so on. ${ }^{54-58}$ However, the optimal pathway of FMT remains undefined. Retention enema is the most common method in China because it is inexpensive and safe, but the ability of microbiota colonization is limited. Oral capsule administration is noninvasive and accepted by patients, but it is cost prohibitive. In addition, it is worth noting that important data should be collect when conducting FMT trials including macro and micronutrient information, such as PPI use, metformin, diabetes status, obesity, antibiotic use, previous GI surgery, IBD, other intestinal diseases ${ }^{8,49,59-64}$ (-Table 1).

\section{Common Indications, Effectiveness, and Safety}

There is a considerable number of clinical trials aimed at investigating diseases in which FMT along with its

Table 1 Collecting information of macro and micronutrients for trials using FMT

\begin{tabular}{|c|c|c|c|c|}
\hline Disease type & Authors, Year & Study type & Nutrients & Primary outcome \\
\hline $\begin{array}{l}\text { Hepatic } \\
\text { encephalopathy (HE) }\end{array}$ & Bajaj et al ${ }^{8} 2018$ & $\mathrm{RCT}$ & $\begin{array}{l}\text { Lactulose, rifaximin, } \\
\text { metronidazole, } \\
\text { amoxicillin, } \\
\text { ciprofloxacin }\end{array}$ & $\begin{array}{l}\text { Participants were divided into the FMT and no } \\
\text { treatment group. In FMT group, FMT increased } \\
\text { diversity of the intestinal flora, and improved } \\
\text { cognitive dysfunction. }\end{array}$ \\
\hline $\mathrm{HE}$ & Kao et al ${ }^{17} 2015$ & Case report & Lactulose and rifaximin & $\begin{array}{l}\text { This report is the finish first report of treating } \\
\text { HE. In this case, the dramatic clinical } \\
\text { improvements, both subjectively and } \\
\text { objectively, following serial FMT are very } \\
\text { encouraging. }\end{array}$ \\
\hline Liver cirrhosis (LC) & Bajaj et al ${ }^{4} 2018$ & $\mathrm{RCT}$ & $\begin{array}{l}\text { Lactulose, rifaximin, } \\
\text { metronidazole, } \\
\text { amoxicillin, } \\
\text { ciprofloxacin, diabetes }\end{array}$ & $\begin{array}{l}\text { In patients with advanced cirrhosis on lactulose } \\
\text { and rifaximin, FMT restored antibiotic- } \\
\text { associated disruption in microbial diversity and } \\
\text { function. }\end{array}$ \\
\hline LC & Fischer et $\mathrm{al}^{5} 2020$ & $\begin{array}{l}\text { A multicenter } \\
\text { observational study }\end{array}$ & $\begin{array}{l}\text { Clostridioides difficile } \\
\text { infection, IBD, } \\
\text { immunosuppression, } \\
\text { ETOH, PSC, ascites, } \\
\text { hepatic } \\
\text { encephalopathy, } \\
\text { variceal hemorrhage, } \\
\text { lactulose, rifaximin }\end{array}$ & $\begin{array}{l}\text { In all, } 63 \text { CDI patients, } 54 \text { patients ( } 85.7 \% \text { ) were } \\
\text { successful by FMT, and side effect is not } \\
\text { significant. FMT has proven to be a safe therapy. }\end{array}$ \\
\hline $\begin{array}{l}\text { Clostridium difficile } \\
\text { infection (CDI) }\end{array}$ & Kelly et al $^{18} 2014$ & $\begin{array}{l}\text { A multicenter } \\
\text { retrospective series }\end{array}$ & $\begin{array}{l}\text { Vancomycin, } \\
\text { antineoplastic agents, } \\
\text { immunocompromised, } \\
\text { solid organ transplant } \\
\text { recipients, HIV/AIDS }\end{array}$ & $\begin{array}{l}12 \text { patients were treated with FMT, and the } \\
\text { overall cure rate was } 89 \% \text {. Therefore, FMT is also } \\
\text { safe for the treatment of immunocompromised } \\
\text { patients with CDI. }\end{array}$ \\
\hline CDI & Salgia et al ${ }^{6} 2020$ & $\begin{array}{l}\text { Observational } \\
\text { study }\end{array}$ & $\begin{array}{l}\text { Hypertension, } \\
\text { immunosuppression, } \\
\text { cancer, chronic kidney } \\
\text { disease, diabetes }\end{array}$ & $\begin{array}{l}\text { A total of } 201 \text { patients with CDI received FMT. } \\
\text { There was no significant difference between } \\
\text { patients with liver disease and the rest of the } \\
\text { cohort with regard to FMT response } 12 / 14 \\
(87 \%) \text { vs. } 164 / 187(88 \%) \text {. }\end{array}$ \\
\hline Alcoholic hepatitis & Philips et al ${ }^{19} 2017$ & Case report & $\begin{array}{l}\text { Ascites, icterus, } \\
\text { prednisolone }\end{array}$ & $\begin{array}{l}\text { Starting on day } 5 \text { of FMT, the general condition } \\
\text { of patient was improved. This result suggests } \\
\text { the beneficial effects of FMT in a patient with } \\
\text { SAH not responding to steroids. }\end{array}$ \\
\hline Hepatitis B virus & Ren et $\mathrm{al}^{20} 2016$ & $\begin{array}{l}\text { Observational } \\
\text { study }\end{array}$ & $\begin{array}{l}\text { Entecavir, tenofovir } \\
\text { disoproxil fumarate }\end{array}$ & $\begin{array}{l}18 \mathrm{HBV} \text { patients were classified in FMT and } \\
\text { control group. At the end of follow-up, a } \\
\text { significant decline in HBeAg titer was observed } \\
\text { in the FMT arm compared with that at the } \\
\text { baseline. In contrast to that, none of the control } \\
\text { patients achieved HBeAg clearance ( } 0 \text { of } 13 \text { ) at } \\
\text { the conclusion of the study. }\end{array}$ \\
\hline $\begin{array}{l}\text { Nonalcoholic fatty liver } \\
\text { disease }\end{array}$ & Craven et $\mathrm{al}^{21} 2020$ & $\begin{array}{l}\text { RCT, double- } \\
\text { blinded }\end{array}$ & Obesity & $\begin{array}{l}21 \text { NAFLD patients were randomized in two } \\
\text { group. There were no significant changes in } \\
\text { HOMA-IR or hepatic PDFF in patients who } \\
\text { received the allogenic or autologous FMT. }\end{array}$ \\
\hline
\end{tabular}

Abbreviations: CHB, Chronic hepatitis B; ETOH, ethanol; FMT, fecal microbiota transplantation; IBD, inflammatory bowel disease; NAFLD, nonalcoholic fatty liver disease; PDFF, proton density fat fraction; PSC, primary sclerosing cholangitis; SAH, severe alcoholic hepatitis. 
effectiveness and safety would be useful. To date, FMT is recognized as the last-resort therapy for severe CDI. ${ }^{65} \mathrm{In}$ deed, evidence showed that FMT was a safe and effective therapy against CDI for patients with CLD and cirrhosis. ${ }^{59,62}$ In addition, FMT is very effective for the treatment of several types of disease, such as intestinal diseases, liver diseases, and neurological disorders. ${ }^{66-69}$ In most reported cases, FMT was shown to be safe, tolerated and highly effective compared with previous measures of regulating gut microbiota, including prebiotics, probiotics, and antibiotics. ${ }^{53}$

A study reported that among 536 patients with CDI who received FMT therapy, the clinical response rate was up to $87 \%$, and no severe adverse events were observed. ${ }^{70}$ Another study used frozen fecal capsules to treat 20 patients with CDI, resulting in a $90 \%$ response rate after one or two treatment courses without serious adverse effects. The above studies indicate that the administration of FMI in CDI is effective and safe. However, in 2019, two patients with CDI were reported to have suffered from bacteremia after undergoing FMT, and one patient died. These two patients received fecal capsules from the same donor, which indicates that FMT also has some risks. It is therefore necessary to enhance donor screening to limit the transmission of microorganisms that could lead to adverse infections. ${ }^{71}$

Ulcerative colitis (UC), an IBD, is difficult to treat, and remission is difficult to achieve. In a randomized trial with 70 acute UC patients, the remission rate in the FMT group and placebo-controlled group was 24 vs. 5\%, respectively. ${ }^{72}$ Moreover, no significant difference in adverse events was observed between the groups. Therefore, FMT induces remission in a significantly greater percentage of patients with UC than placebo with no additional adverse events. Yu et al evaluated the efficacy of oral FMT capsules in 22 adults with obesity in a double-blind, randomized trial. ${ }^{73}$ The results showed that the capsules were well tolerated and led to sustained changes in the gut microbiome and BA profiles that were similar to those of the lean donor. Nevertheless, FMT did not reduce body mass index (BMI) in obese patients compared with the placebo-controlled group.

In intestinal infectious diseases and intestinal inflammatory diseases, there is abundant evidence proving the efficacy of FMT. In metabolic diseases, FMT could improve lipid metabolism. With strict donor screening, FMT could be quite safe. Many studies have reported its remarkable effect in various diseases; therefore, the clinical indications of FMT could be further expanded to include other diseases, such as liver disease.

However, FMT safety still continues to be a significant problem when FMT is considered a treatment option. Side effects mainly included diarrhea, constipation, abdominal pain, and low-grade fevers. ${ }^{74}$ Though generally safe, rare but serious adverse events form FMT, including death, have been reported. Other than that, the infectious agent is also a major cause of serious adverse events, such as bacteremia and infection. ${ }^{74}$ So donor screening is very important to prevent pathogen transmission through stool.

\section{Fecal Microbiota Transplantation to Treat Microbiota Imbalances in Liver Disease}

\section{Hepatic Encephalopathy}

$\mathrm{HE}$ is the most serious complication of liver cirrhosis. Recurrent $\mathrm{HE}$ is a leading cause of readmission, brain injury, and death. Upper gastrointestinal bleeding and serious infection trigger recurrence in many of these patients. They commonly receive antibiotic therapy, which could disrupt gut microbiota, precipitating further HE. The standard care of HE, including lactulose and rifaximin, could improve the environment of the microbiota in the gut. However, their therapeutic effect is relatively poor.

Few studies analyzing the efficacy of FMT in HE have been published to date. In 2016, Kao et al reported the first case of grade 1-2 HE who underwent FMT. ${ }^{61}$ The results of serial FMT have been very encouraging. Both subjectively and objectively, the symptoms of the patients improved. Later, an increasing number of researchers begun to explore FMT in HE. A study exploring the effect of capsular FMT in HE patients tested different indexes, including safety, tolerance, microbiota changes, and brain function. After FMT treatment, duodenal mucosal diversity, duodenal E-cadherin, and defense A5 were increased, while IL-6 and serum LBP were reduced. ${ }^{75}$ Mehta et al $^{9}$ shared their experience in treating 10 HE patients with FMT. Their results showed that arterial ammonia concentration decreased significantly, and neurological symptoms were reduced at 20 weeks after receiving FMT. In an open-label randomized clinical trial, Bajaj et $\mathrm{al}^{8}$ conducted a study on cirrhosis with recurrent HE patients, comparing the effect and safety between FMT using a rationally derived stool donor and the standard of care (SOC) alone. The results showed that the adverse events and incidence rate of recurrent HE in the FMT group were significantly lower than those in the SOC group. The bacterial diversity and beneficial taxa were increased in the FMT group but not in the SOC group. More importantly, cognition was significantly improved in the FMT group. Therefore, FMT was associated with lower hospitalizations, improved cognition, and balanced gut microbiota among patents with HE.

In addition to clinical studies, preclinical studies also support the application of FMT in HE. To investigate whether FMT could prevent the occurrence of $\mathrm{HE}$, Wang et $\mathrm{al}^{76}$ established an HE rat model with carbon tetrachloride (CCl4)-induced acute hepatic dysfunction. The rats subsequently received FMT, and the results revealed that FMT could improve rat behaviors, HE grade, and spatial learning capability. Moreover, FMT prevented hepatic necrosis and intestinal mucosal barrier damage. FMT may alter intestinal permeability and improve the TLR response of the liver by decreasing TLR4 and TLR9 expression. In conclusion, both preclinical and clinical evidence suggest that FMT can improve the prognosis of HE patients.

\section{Complications of Liver Cirrhosis}

Gut dysbiosis appears in 20 to $75 \%$ of patients with cirrhosis. The microbiota composition and barrier function are affected, even with prior rifaximin use. Moreover, the incidence of 
cirrhosis and its complications have been linked to abnormalities in the gut microbiota. ${ }^{77}$ Common complications of liver cirrhosis include spontaneous bacterial peritonitis, ascites, and sepsis. In patients with cirrhosis, a complex and specific microbiota composition has been detected, and Clostridiales is increased in the serum of patients with ascites. $^{78}$ Importantly, BT is increased in the presence of portal hypertension. These changes in function and structure are mainly related to fecal BAs and short-chain fatty acids (SCFAs). The imbalance of gut microbiota, which worsens with the progression of liver disease, is further impaired by the administration of antibiotics. Restoring the gut microbiota could therefore both decrease ammonia serum and endotoxemia levels and prevent complications of cirrhosis.

A phase I safety trial was performed to explore FMT in restoring gut microbial function in cirrhosis, with 10 patients assigned to FMT and SOC groups. ${ }^{49}$ In the background of antibiotics and rifaximin, the microbial composition and functional indicators (BA, SCFA, $7 \alpha$-dehydroxylation) were analyzed before and after a single FMT in this study. The researchers found that FMT could induce the secretion of SCFAs and modulate BA profiles and thus restore metabolic capability, especially in the short term after FMT. The longterm effects of FMT require further exploration. Because of the limited sample sizes of the current studies in the literature, more prospective, randomized and large-scale studies are needed to further confirm the value of FMT in cirrhosis.

\section{Primary Sclerosing Cholangitis}

PSC is a chronic autoimmune liver disease with a poor prognosis and is often associated with IBD. Liver transplantation remains the only curative option for these patients. It has been proven that the imbalance of gut microbiota is related to the progression of PSC. A GF murine model of PSC proved that the absence of the gut microbiota exacerbated hepatobiliary diseases, explaining the importance of FMT from another perspective. ${ }^{79}$ Microbiome restoration has become a therapeutic goal of PSC due to the lack of effective medical therapy. ${ }^{80}$ Previous studies have shown that oral vancomycin could be effective in treating PSC patients by modifying the gut microbiome. As such, FMT may be a more feasible treatment for PSC. FMT could improve microbiome diversity and liver biochemistry in PSCs.

The first case report of FMT treatment for PSC was published in 2018 by Philips. ${ }^{81}$ A 38-year-old male was diagnosed with PSC with recurrent acute bacterial cholangitis and without IBD. The patient underwent weekly endoscopic FMT for 4 weeks, and all antibiotics were withheld during this period. Multiple studies have shown that a decrease in BAs and alkaline phosphatase (ALP) lead to a better prognosis. After treatment with FMT, the patient's BAs and ALP decreased, and the patient remained anicteric up to a year. Regarding this gut microbiota, a decrease in Proteobacteria (a phylum containing numerous human pathogens) and an increase in Firmicutes abundance (a phylum with many taxa, having presumed beneficial immunologic properties) were observed. All of these results favored the use of FMT in PSC.
In 2019, Allegretti et $\mathrm{a}^{82}$ enrolled 10 patients with PSC and IBD. All of them received a single FMT from a single donor by colonoscopy, and there were no related adverse events. ALP levels decreased $\geq 50 \%$ from baseline in $30 \%$ of patients, and the diversity of microbiota and metabonomic dynamics increased after FMT. More importantly, the increase in bacterial diversity and engraftment may correlate with an improvement in ALP. This was the first prospective study to report the efficacy and safety of FMT in PSC.

The results from these studies suggest that FMT is safe for treating PSC, although the ability of FMT to improve the disease and the courses of FMT have not yet been determined. Some studies administered four fecal transplants, while others used a single transplant. Exploring optimal FMT treatment courses and time points is important for determining the efficacy of FMT in PSC.

\section{Fatty Liver (Alcoholic, Nonalcoholic)}

Patients with severe alcoholic hepatitis (SAH) have a very poor prognosis, with a 28-day mortality rate of 13 to $30 \%$ and a 1-year mortality/need for liver transplant of nearly $60 \%$. After alcohol consumption stops and lifestyle changes are made, fatty liver continues to develop. Thus, treatment options are severely restricted. It has been described that the progression of both ALD and NAFLD is associated with an imbalance in gut microbiota. Limited clinical studies have evaluated the efficacy of FMT in ALD and NAFLD.

Philips et $a^{83}$ conducted a pilot study with eight male patients to investigate the effect of FMT in untreated SAH. Daily FMT was given to the participants for 1 week. They found that FMT treatment was effective and safe, with a reduction in both disease severity and mortality. The oneyear survival rate was $87.5 \%$, while it was $33.3 \%$ in the control group. A large number of retrospective studies showed similarly encouraging results. Fifty-one patients received steroids, nutritional therapy, pentoxifylline, and FMT, and the survival rates were $38,29,30$, and $75 \%$, respectively, after 3 months. Patients in the FMT group had a significantly higher survival rate and decreased rates of HE than other groups. ${ }^{84}$ While the above studies showed that FMT is promising for the treatment of $\mathrm{SAH}$, they are all retrospective studies. The results of a prospective and randomized study comparing FMT and corticosteroid therapy for SAH (NCT03091010) have not yet been published. Recent studies suggest that Virome and Fungome are altered in patients with alcoholic hepatitis and represent novel targets for therapy. For instance, Schnabl et al also found that an intestinal Virome signature was associated with disease severity and mortality. ${ }^{85}$ In the field of fungi research, the latest research found that alcoholism can significantly alter intestinal fungal diversity and composition: the abundance of Candida in ALD patients was remarkably lower; however, that of penicillium in nonalcoholic controls was higher. ${ }^{86}$

FMT was used in a study comparing 64 obese children with sonographic NAFLD in a randomized triple-blind trial. After receiving probiotic capsules, the levels of alanine aminotransferase, aspartate aminotransferase, cholesterol, and triglycerides and waist circumference decreased 
significantly, indicating that probiotic compounds were effective at treating pediatric NAFLD. ${ }^{87}$ Probiotic capsules are also effective in NAFLD. Therefore, FMT, which has more beneficial bacteria and a normal gut microbiota structure, should be even more effective. Zhou et al ${ }^{88}$ investigated the role of FMT in curing steatohepatitis in mice. Their results revealed that FMT could attenuate steatohepatitis by correcting gut microbiota disturbances and decreasing intrahepatic lipid accumulation, proinflammatory cytokines, and NAS scores. Prof. Perlemuter transplanted the intestinal microflora from ALD patients who have AH into germ-free mice, which lead to severe alcohol-induced liver inflammation. ${ }^{14}$ Also, Cassard et al found that pectin treatment and FMT both prevented alcohol-induced liver injury. ${ }^{89}$ These studies have convincingly demonstrated that FMT will be adopted into preclinical practice with ALD. However, there are relatively few clinical studies exploring FMT in chronic ALD and NAFLD. Further studies on FMT in fatty liver diseases are certainly warranted.

\section{Acute Liver Injury}

ALI is defined as acute hepatocyte damage and necrosis because of different factors, including alcohol, paracetamol toxicity, hepatic ischemia, viral and autoimmune hepatitis, and drug-induced liver injury. If patients are not promptly diagnosed and expeditiously treated, ALI may develop acute liver failure (ALF), with a significantly high mortality of up to $40 \%$ within 90 days. More than half of the cases of ALF progress and require liver transplantation. An excessive systemic inflammatory response seems to play a crucial role in the development of ALI and ALF. The imbalance in the gut microbiota is related not only to the systemic inflammatory response but also to ALI itself. For example, Lactobacillus salivarius LI01 was reported to attenuate hepatic injury. ${ }^{90}$ During the treatment of ALI or ALF, the use of probiotics is commonly recognized, but it brings limited survival benefits. FMT is a potential treatment strategy for ALI and ALF to regulate the gut microbiota.

HE is the leading cause of death in ALF. A rat model of ALF was established with $\mathrm{CCl} 4$, and FMT was found to have potent protective effects on motor activity in the rat model that were comparable to probiotic administration. FMT effectively improves the behavior and spatial cognitive capabilities of HE. More importantly, biochemical indicators of hepatotoxicity (ALT, AST, ALP, TBIL, DTBIL) and inflammatory mediators (IL-1 $\beta$, TNF- $\alpha$, TLR4, TLR9) were significantly reduced in the FMT group compared with the control group. At the same time, decreased intestinal permeability and serum ammonia were observed in the FMT rat model. In conclusion, FMT prevented hepatic necrosis in ALI by decreasing intestinal permeability, clearing ammonia, and limiting systemic inflammation. ${ }^{76}$ These animal studies have provided a basis for the clinical application of FMT in ALT and ALF.

\section{Future Prospects}

Hepatitis B virus (HBV) infection continues to be a serious global disease burden, especially in China, with an incidence rate of up to 7 to $8 \%$. If the infection is not treated, it may transform into liver cirrhosis or liver cancer. Therefore, the management of HBV infection is critical to decrease the incidence of cirrhosis and liver cancer. By comparing the intestinal flora of CHB patients and healthy controls, Wang et al revealed that the bacteroides level was reduced in $\mathrm{CHB}$ patients based on sequencing the V3-V4 region of the $16 \mathrm{~S}$ rRNA gene of the gut microbiota. In addition, the structural changes in the gut microbiota due to liver disease and the severity of disease are mutually causal. They probably affect the transformation from $\mathrm{CHB}$ to liver fibrosis, liver cancer or liver failure. Mouse studies have also demonstrated that the gut microbiota may influence the host's immune response and ability to clear HBV infection. Therefore, FMT could be considered a potential immunomodulatory therapy for chronic infection with HBV.

In a nonrandomized controlled study, $14 \mathrm{HBeAg}$-positive patients were given six cycles of FMT via gastroscopy accompanied by antiviral therapy (ART); 16.7\% (2/12) of these patients exhibited HBeAg clearance in the FMT arm, whereas none exhibited clearance in the ART arm, compared with 15 patients with ART alone. ${ }^{91}$ Another study with 18 HBeAgpositive patients reported similar results. ${ }^{64}$ In the FMT arm, two of five patients exhibited HBeAg clearance, compared with none of the 13 patients in the control arm. These findings indicate that FMT is effective against $\mathrm{HBC}$ infection. However, the long-term effects of FMT on chronic HBC infection remain unclear. In-depth research is required to obtain solid conclusions regarding FMT in hepatitis.

Hepatocellular carcinoma (HCC) is the third leading cause of cancer death globally and is strongly associated with the development of HBV and hepatitis $\mathrm{C}$ virus infection. Experiments in mice found that imbalance of gut microbiota increased the incidence rate of $\mathrm{HCC},{ }^{92}$ and tumor growth can be inhibited by administration of probiotics. ${ }^{93}$ These studies suggest that modification of the gut microbiota may improve the results of HCC patients. FMT has huge potential for application in the management of HCC. However, there is no clinical research reporting on FMT in the treatment of HCC to date, which warrants further investigation. The current challenges and ongoing trials testing MFT in patients with liver disease such as HBV and HCC can be found in clincialtrials.gov as summarized in - Table 2 .

\section{Conclusion}

The imbalance of gut microbiota contributes significantly to various liver diseases. FMT offers a novel approach to treat liver diseases by restoring the healthy gut microbiota, reducing the incidence and mortality of ALI or HE and improving the survival rate of PSC. Compared with previous conventional methods (probiotics), FMT is the most direct way to change the composition of gut microbiota. Both basic science research and clinical studies have proven that FMT can effectively improve the state of the gut microbiota under the conditions of acute and chronic liver diseases, rebuild the intestinal microbiome balance, and accelerate disease recovery. 


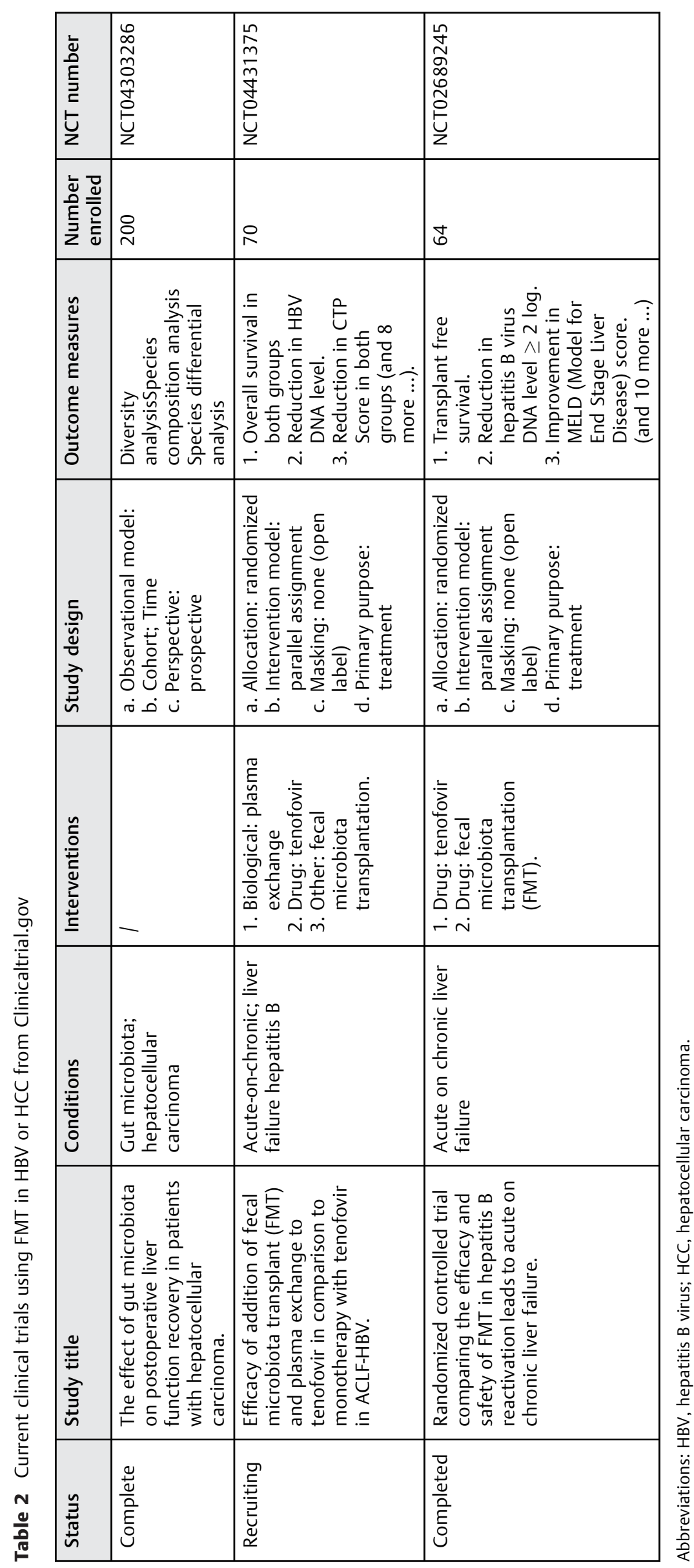


However, only a few clinical studies have investigated FMT for the treatment of chronic hepatitis B and liver HCC. Large randomized controlled studies are urgently needed to explore the effectiveness of FMT in hepatitis and HCC, especially its long-term efficacy, to reduce the disease burden in our country.

Although FMT is an effective, safe, and inexpensive treatment approach for many diseases, it also has several limitations, such as acceptance by patients. The promotion of FMT should therefore be strengthened. Additionally, transplantation methods need to be further improved. It is urgent to build a fecal bacteria bank to provide a source of healthy fecal bacteria for patients.

\section{Main Concepts and Learning Points}

- Gut microbiota abnormalities affect acute liver injury.

- Fecal microbiota transplantation (FMT) could contribute to clinical efficacy in hepatic disorders.

- FMT could be considered as a potential immunomodulatory therapy for with HBV.

\section{Authors' Contributions}

X.G. and L.C. designed the concept of the study. Q.L., C.Z., and X.G. wrote the first draft of the manuscript. L.C. and Z. $\mathrm{T}$. edited the subsequent versions of the manuscript.

\section{Funding}

National Natural Science Foundation of China (81904129), the College Student Innovation Funds Project for the Hebei University of Chinese Medicine (31800952, 202014432203), the Fundamental Research Funds for the Southeast University (3218006405, 2242019s10024) sponsored this study.

\section{Conflict of Interest}

None declared.

\section{References}

1 Medini D, Serruto D, Parkhill J, et al. Microbiology in the postgenomic era. Nat Rev Microbiol 2008;6(06):419-430

2 Clemente JC, Ursell LK, Parfrey LW, Knight R. The impact of the gut microbiota on human health: an integrative view. Cell 2012;148 (06):1258-1270

3 Palacios T, Vitetta L, Coulson S, et al. Targeting the intestinal microbiota to prevent type 2 diabetes and enhance the effect of metformin on glycaemia: a randomised controlled pilot study. Nutrients 2020;12(07):12

4 van Wietmarschen HA, Busch M, van Oostveen A, Pot G, Jong MC. Probiotics use for antibiotic-associated diarrhea: a pragmatic participatory evaluation in nursing homes. BMC Gastroenterol 2020;20(01):151

5 Bakken JS, Borody T, Brandt LJ, et al; Fecal Microbiota Transplantation Workgroup. Treating Clostridium difficile infection with fecal microbiota transplantation. Clin Gastroenterol Hepatol 2011;9(12):1044-1049

6 Betrapally NS, Gillevet PM, Bajaj JS. Gut microbiome and liver disease. Transl Res 2017;179:49-59

7 Hawkins KG, Casolaro C, Brown JA, Edwards DA, Wikswo JP. The microbiome and the gut-liver-brain axis for central nervous system clinical pharmacology: challenges in specifying and integrating in vitro and in silico models. Clin Pharmacol Ther 2020; 108(05):929-948

8 Bajaj JS, Kassam Z, Fagan A, et al. Fecal microbiota transplant from a rational stool donor improves hepatic encephalopathy: a randomized clinical trial. Hepatology 2017;66(06):1727-1738

9 Mehta R, Kabrawala M, Nandwani S, et al. Preliminary experience with single fecal microbiota transplant for treatment of recurrent overt hepatic encephalopathy-a case series. Indian J Gastroenterol 2018;37(06):559-562

10 Le Roy T, Llopis M, Lepage P, et al. Intestinal microbiota determines development of non-alcoholic fatty liver disease in mice. Gut 2013;62(12):1787-1794

11 Gomez-Hurtado I, Gallego-Duran R, Zapater P, et al. Bacterial antigen translocation and age as BMI-independent contributing factors on systemic inflammation in NAFLD patients. Liver Int 2020;40(09):2182-2193

12 Frank DN, Zhu W, Sartor RB, Li E. Investigating the biological and clinical significance of human dysbioses. Trends Microbiol 2011; 19(09):427-434

13 Boursier J, Diehl AM. Implication of gut microbiota in nonalcoholic fatty liver disease. PLoS Pathog 2015;11(01): e1004559

14 Llopis M, Cassard AM, Wrzosek L, et al. Intestinal microbiota contributes to individual susceptibility to alcoholic liver disease. Gut 2016;65(05):830-839

15 Furukawa M, Moriya K, Nakayama J, et al. Gut dysbiosis associated with clinical prognosis of patients with primary biliary cholangitis. Hepatol Res 2020;50(07):840-852

16 Wu G, Win S, Than TA, Chen P, Kaplowitz N. Gut microbiota and liver injury (I)-acute liver injury. Adv Exp Med Biol 2020; 1238:23-37

17 Wang Y, Pan CQ Xing H. Advances in gut microbiota of viral hepatitis cirrhosis. BioMed Res Int 2019;2019:9726786

18 Alonso C, Fernández-Ramos D, Varela-Rey M, et al. Metabolomic identification of subtypes of nonalcoholic steatohepatitis. Gastroenterology 2017;152(06):1449-1461.e7

19 Kaikkonen JE, Würtz P, Suomela E, et al. Metabolic profiling of fatty liver in young and middle-aged adults: cross-sectional and prospective analyses of the Young Finns Study. Hepatology 2017; 65(02):491-500

20 Loomba R, Seguritan V, Li W, et al. Gut microbiome-based metagenomic signature for non-invasive detection of advanced fibrosis in human nonalcoholic fatty liver disease. Cell Metab 2017;25(05):1054-1062.e5

21 Boursier J, Mueller O, Barret M, et al. The severity of nonalcoholic fatty liver disease is associated with gut dysbiosis and shift in the metabolic function of the gut microbiota. Hepatology 2016;63 (03):764-775

22 Oh TG, Kim SM, Caussy C, et al. A universal gut-microbiomederived signature predicts cirrhosis. Cell Metab 2020;32(05): 878-888.e6

23 Oh TG, Kim SM, Atkins AR, et al. Proton pump inhibitor use status does not modify the microbiome signature for cirrhosis. Cell Metab 2021;33(03):457

24 Aron-Wisnewsky J, Vigliotti C, Witjes J, et al. Gut microbiota and human NAFLD: disentangling microbial signatures from metabolic disorders. Nat Rev Gastroenterol Hepatol 2020;17(05): 279-297

25 Adams LA, Wang Z, Liddle C, et al. Bile acids associate with specific gut microbiota, low-level alcohol consumption and liver fibrosis in patients with non-alcoholic fatty liver disease. Liver Int 2020; 40(06):1356-1365

26 Ghoshal UC, Goel A, Quigley EMM. Gut microbiota abnormalities, small intestinal bacterial overgrowth, and non-alcoholic fatty liver disease: an emerging paradigm. Indian J Gastroenterol 2020;39(01):9-21 
27 Guohong-Liu, Qingxi-Zhao, Hongyun-Wei. Characteristics of intestinal bacteria with fatty liver diseases and cirrhosis. Ann Hepatol 2019;18(06):796-803

28 Shah A, Macdonald GA, Morrison M, Holtmann G. Targeting the gut microbiome as a treatment for primary sclerosing cholangitis: a conceptional framework. Am J Gastroenterol 2020;115(06):814-822

29 Buness JG, Ali AH, Tabibian JH, Buness CW, Cox KL, Lindor KD. Potential association of doxycycline with the onset of primary sclerosing cholangitis: a case series. Am J Ther 2019;26(04): e551-e557. Doi: 10.1097/MJT.0000000000001065

30 Liwinski T, Zenouzi R, John C, et al. Alterations of the bile microbiome in primary sclerosing cholangitis. Gut 2020;69(04):665-672

31 Chen Z, Xie Y, Zhou F, et al. Featured gut microbiomes associated with the progression of chronic hepatitis B disease. Front Microbiol 2020;11:383

32 Beraza N. Fibrosis and the intestinal microbiome; a focus on chronic liver disease. Curr Opin Pharmacol 2019;49:76-81

33 Jia W, Rajani C, Kaddurah-Daouk R, Li H. Expert insights: the potential role of the gut microbiome-bile acid-brain axis in the development and progression of Alzheimer's disease and hepatic encephalopathy. Med Res Rev 2020;40(04):1496-1507

34 Liu R, Kang JD, Sartor RB, et al. Neuroinflammation in murine cirrhosis is dependent on the gut microbiome and is attenuated by fecal transplant. Hepatology 2020;71(02):611-626

35 Bajaj JS, Fagan A, White MB, et al. Specific gut and salivary microbiota patterns are linked with different cognitive testing strategies in minimal hepatic encephalopathy. Am J Gastroenterol 2019;114(07):1080-1090

36 Acharya C, Bajaj JS. Current management of hepatic encephalopathy. Am J Gastroenterol 2018;113(11):1600-1612

37 Bajaj JS, Ridlon JM, Hylemon PB, et al. Linkage of gut microbiome with cognition in hepatic encephalopathy. Am J Physiol Gastrointest Liver Physiol 2012;302(01):G168-G175

38 Lin RS, Lee FY, Lee SD, et al. Endotoxemia in patients with chronic liver diseases: relationship to severity of liver diseases, presence of esophageal varices, and hyperdynamic circulation. J Hepatol 1995;22(02):165-172

39 Wang R, Tang R, Li B, Ma X, Schnabl B, Tilg H. Gut microbiome, liver immunology, and liver diseases. Cell Mol Immunol 2021;18(01): 4-17

40 Cesaro C, Tiso A, Del Prete A, et al. Gut microbiota and probiotics in chronic liver diseases. Dig Liver Dis 2011;43(06):431-438

$41 \mathrm{Mu}$ J, Tan F, Zhou X, Zhao X. Lactobacillus fermentum CQPC06 in naturally fermented pickles prevents non-alcoholic fatty liver disease by stabilizing the gut-liver axis in mice. Food Funct 2020; 11(10):8707-8723

42 Acharya C, Bajaj JS. Altered microbiome in patients with cirrhosis and complications. Clin Gastroenterol Hepatol 2019;17(02): 307-321

43 Lee NY, Suk KT. The role of the gut microbiome in liver cirrhosis treatment. Int J Mol Sci 2020;22(01):16

44 Kang DJ, Betrapally NS, Ghosh SA, et al. Gut microbiota drive the development of neuroinflammatory response in cirrhosis in mice. Hepatology 2016;64(04):1232-1248

45 Caussy C, Hsu C, Lo M-T, et al; Genetics of NAFLD in Twins Consortium. Link between gut-microbiome derived metabolite and shared gene-effects with hepatic steatosis and fibrosis in NAFLD. Hepatology 2018;68(03):918-932

46 Trapecar M, Communal C, Velazquez J, et al. Gut-liver physiomimetics reveal paradoxical modulation of IBD-related inflammation by short-chain fatty acids. Cell Syst 2020;10(03):223-239.e9

47 Saeedi BJ, Liu KH, Owens JA, et al. Gut-resident lactobacilli activate hepatic Nrf2 and protect against oxidative liver injury. Cell Metab 2020;31(05):956-968.e5

48 Woodhouse CA, Patel VC, Goldenberg S, et al. PROFIT, a PROspective, randomised placebo controlled feasibility trial of Faecal mIcrobiota Transplantation in cirrhosis: study protocol for a single-blinded trial. BMJ Open 2019;9(02):e023518
49 Bajaj JS, Kakiyama G, Savidge T, et al. Antibiotic-associated disruption of microbiota composition and function in cirrhosis is restored by fecal transplant. Hepatology 2018;68(04): $1549-1558$

50 Zhang F, Luo W, Shi Y, Fan Z, Ji G. Should we standardize the 1,700year-old fecal microbiota transplantation? Am J Gastroenterol 2012;107(11):1755, author reply 1755-1756

51 Eiseman B, Silen W, Bascom GS, Kauvar AJ. Fecal enema as an adjunct in the treatment of pseudomembranous enterocolitis. Surgery 1958;44(05):854-859

52 Schwan A, Sjölin S, Trottestam U, Aronsson B. Relapsing clostridium difficile enterocolitis cured by rectal infusion of homologous faeces. Lancet 1983;2(8354):845

53 Kelly CR, Kahn S, Kashyap P, et al. Update on fecal microbiota transplantation 2015: indications, methodologies, mechanisms, and outlook. Gastroenterology 2015;149(01): 223-237

54 Youngster I, Russell GH, Pindar C, Ziv-Baran T, Sauk J, Hohmann EL. Oral, capsulized, frozen fecal microbiota transplantation for relapsing Clostridium difficile infection. JAMA 2014;312(17): 1772-1778

55 Gustafsson A, Berstad A, Lund-Tønnesen S, Midtvedt T, Norin E. The effect of faecal enema on five microflora-associated characteristics in patients with antibiotic-associated diarrhoea. Scand J Gastroenterol 1999;34(06):580-586

56 Persky SE, Brandt LJ. Treatment of recurrent Clostridium difficileassociated diarrhea by administration of donated stool directly through a colonoscope. Am J Gastroenterol 2000;95(11): 3283-3285

57 Yin G, Li JF, Sun YF, et al. [Fecal microbiota transplantation as a novel therapy for severe psoriasis]. Zhonghua Nei Ke Za Zhi 2019; 58(10):782-785

58 Liu J, Miyake H, Zhu H, et al. Fecal microbiota transplantation by enema reduces intestinal injury in experimental necrotizing enterocolitis. J Pediatr Surg 2020;55(06):1094-1098

59 Cheng Y-W, Alhaffar D, Saha S, et al. Fecal microbiota transplantation is safe and effective in patients with Clostridioides difficile infection and cirrhosis. Clin Gastroenterol Hepatol 2020;18(08): 1874-1881. Doi: 10.1016/j.cgh.2020.06.051

60 Craven L, Rahman A, Nair Parvathy S, et al. Allogenic fecal microbiota transplantation in patients with nonalcoholic fatty liver disease improves abnormal small intestinal permeability: a randomized control trial. Am J Gastroenterol 2020;115(07): 1055-1065

61 Kao D, Roach B, Park H, et al. Fecal microbiota transplantation in the management of hepatic encephalopathy. Hepatology 2016;63 (01):339-340

62 Meighani A, Alimirah M, Ramesh M, Salgia R. Fecal microbiota transplantation for Clostridioides difficile infection in patients with chronic liver disease. Int J Hepatol 2020;2020:1874570

63 Philips CA, Phadke N, Ganesan K, Augustine P. Healthy donor faecal transplant for corticosteroid non-responsive severe alcoholic hepatitis. BMJ Case Rep 2017;2017:bcr-2017222310

64 Ren YD, Ye ZS, Yang LZ, et al. Fecal microbiota transplantation induces hepatitis B virus e-antigen ( $\mathrm{HBeAg}$ ) clearance in patients with positive $\mathrm{HBeAg}$ after long-term antiviral therapy. Hepatology 2017;65(05):1765-1768

65 Surawicz CM, Brandt LJ, Binion DG, et al. Guidelines for diagnosis, treatment, and prevention of Clostridium difficile infections. Am J Gastroenterol 2013;108(04):478-498, quiz 499

66 Vaughn BP, Rank KM, Khoruts A. Fecal microbiota transplantation: current status in treatment of GI and liver disease. Clin Gastroenterol Hepatol 2019;17(02):353-361

67 Vendrik KEW, Ooijevaar RE, de Jong PRC, et al. Fecal microbiota transplantation in neurological disorders. Front Cell Infect Microbiol 2020;10:98 
68 Gerussi A, Lucà M, Cristoferi L, et al. New therapeutic targets in autoimmune cholangiopathies. Front Med (Lausanne) 2020; $7: 117$

69 Mahajan R, Midha V, Singh A, et al. Incidental benefits after fecal microbiota transplant for ulcerative colitis. Intest Res 2020;18 (03):337-340

70 Cammarota G, Ianiro G, Gasbarrini A. Fecal microbiota transplantation for the treatment of Clostridium difficile infection: a systematic review. J Clin Gastroenterol 2014;48(08):693-702

71 DeFilipp Z, Bloom PP, Torres Soto M, et al. Drug-Resistant E. coli bacteremia transmitted by fecal microbiota transplant. N Engl J Med 2019;381(21):2043-2050

72 Moayyedi P, Surette MG, Kim PT, et al. Fecal microbiota transplantation induces remission in patients with active ulcerative colitis in a randomized controlled trial. Gastroenterology 2015; 149(01):102-109.e6

73 Yu EW, Gao L, Stastka P, et al. Fecal microbiota transplantation for the improvement of metabolism in obesity: the FMT-TRIM double-blind placebo-controlled pilot trial. PLoS Med 2020;17(03): e1003051

74 Dailey FE, Turse EP, Daglilar E, Tahan V. The dirty aspects of fecal microbiota transplantation: a review of its adverse effects and complications. Curr Opin Pharmacol 2019;49:29-33

75 Bajaj JS, Salzman NH, Acharya C, et al. Fecal microbial transplant capsules are safe in hepatic encephalopathy: a phase 1, randomized, placebo-controlled trial. Hepatology 2019;70(05):1690-1703

76 Wang WW, Zhang Y, Huang XB, You N, Zheng L, Li J. Fecal microbiota transplantation prevents hepatic encephalopathy in rats with carbon tetrachloride-induced acute hepatic dysfunction. World J Gastroenterol 2017;23(38):6983-6994

77 Lachar J, Bajaj JS. Changes in the microbiome in cirrhosis and relationship to complications: hepatic encephalopathy, spontaneous bacterial peritonitis, and sepsis. Semin Liver Dis 2016;36 (04):327-330

78 Santiago A, Pozuelo M, Poca M, et al. Alteration of the serum microbiome composition in cirrhotic patients with ascites. Sci Rep 2016;6:25001

79 Tabibian JH, O'Hara SP, Trussoni CE, et al. Absence of the intestinal microbiota exacerbates hepatobiliary disease in a murine model of primary sclerosing cholangitis. Hepatology 2016;63(01):185-196

80 Bajaj JS, Hays RA. Manipulation of the gut-liver axis using microbiome restoration therapy in primary sclerosing cholangitis. Am J Gastroenterol 2019;114(07):1027-1029

81 Philips CA, Augustine P, Phadke N. Healthy donor fecal microbiota transplantation for recurrent bacterial cholangitis in primary sclerosing cholangitis-a single case report. J Clin Transl Hepatol 2018;6(04):438-441

82 Allegretti JR, Kassam Z, Carrellas M, et al. Fecal microbiota transplantation in patients with primary sclerosing cholangitis: a pilot clinical trial. Am J Gastroenterol 2019;114(07): 1071-1079

83 Philips CA, Pande A, Shasthry SM, et al. healthy donor fecal microbiota transplantation in steroid-ineligible severe alcoholic hepatitis: a pilot study. Clin Gastroenterol Hepatol 2017;15(04): 600-602

84 Philips CA, Phadke N, Ganesan K, Ranade S, Augustine P. Corticosteroids, nutrition, pentoxifylline, or fecal microbiota transplantation for severe alcoholic hepatitis. Indian J Gastroenterol 2018; 37(03):215-225

85 Jiang L, Lang SJ, Duan Y, et al. Intestinal virome in patients with alcoholic hepatitis. Hepatology 2020;72(06):2182-2196

86 Lang S, Duan Y, Liu J, et al. Intestinal fungal dysbiosis and systemic immune response to fungi in patients with alcoholic hepatitis. Hepatology 2020;71(02):522-538

87 Famouri F, Shariat Z, Hashemipour M, Keikha M, Kelishadi R. Effects of probiotics on nonalcoholic fatty liver disease in obese children and adolescents. J Pediatr Gastroenterol Nutr 2017;64 (03):413-417

88 Zhou D, Pan Q, Shen F, et al. Total fecal microbiota transplantation alleviates high-fat diet-induced steatohepatitis in mice via beneficial regulation of gut microbiota. Sci Rep 2017;7(01):1529

89 Ferrere G, Wrzosek L, Cailleux F, et al. Fecal microbiota manipulation prevents dysbiosis and alcohol-induced liver injury in mice. J Hepatol 2017;66(04):806-815

90 Zhuge A, Li B, Yuan Y, et al. Lactobacillus salivarius LI01 encapsulated in alginate-pectin microgels ameliorates D-galactosamineinduced acute liver injury in rats. Appl Microbiol Biotechnol 2020; 104(17):7437-7455

91 Chauhan A, Kumar R, Sharma S, et al. Fecal microbiota transplantation in hepatitis B e antigen-positive chronic hepatitis B patients: a pilot study. Dig Dis Sci 2021;66(03):873-880

92 Ronis MJ, Mercer KE, Shankar K, et al. Potential role of gut microbiota, the proto-oncogene PIKE (Agap2) and cytochrome P450 CYP2W1 in promotion of liver cancer by alcoholic and nonalcoholic fatty liver disease and protection by dietary soy protein. Chem Biol Interact 2020;325:109131

93 Behzadi E, Mahmoodzadeh Hosseini H, Imani Fooladi AA. The inhibitory impacts of Lactobacillus rhamnosus GG-derived extracellular vesicles on the growth of hepatic cancer cells. Microb Pathog 2017;110:1-6

94 Chu L, Huang Y, Xu Y, et al. An LC-APCI+-MS/MS-based method for determining the concentration of neurosteroids in the brain of male mice with different gut microbiota. Journal of Neuroscience Mehods 2021;360:109268

95 Chu LX, Li N, Deng J, et al. LC-APCl+-MS/MS method for the analysis of ten hormones and two endocannabinoids in plasma and hair from the mice with different gut microbiota. J Pharm Biomed Anal 2020;185:113223 